\title{
Evolution of Neural Networks for Physically Simulated Evolved Virtual Quadruped Creatures
}

\author{
Neil Vaughan ${ }^{1,2}$ \\ ${ }^{1}$ University of Chester, UK \\ ${ }^{2}$ Royal Academy of Engineering, London, UK \\ n. vaughanechester.ac.uk
}

\begin{abstract}
This work develops evolved virtual creatures (EVCs) using neuroevolution as the controller for movement and decisions within a 3D physics simulated environment. Previous work on EVCs has displayed various behaviour such as following a light source. This work is focused on complexifying the range of behaviours available to EVCs. This work uses neuroevolution for learning specific actions combined with other controllers for making higher level decisions about which action to take in a given scenario. Results include analysis of performance of the EVCs in simulated physics environment. Various controllers are compared including a hard coded benchmark, a fixed topology feed forward artificial neural network and an evolving ANN subjected to neuroevolution by applying mutations in both topology and weights. The findings showed that both fixed topology ANNs and neuroevolution did successfully control the evolved virtual creatures in the distance travelling task.
\end{abstract}

Keywords: Evolved Creatures, Neuroevolution, Quadruped gait.

\section{Introduction}

Since the pioneering work of a computer artist Karl Sims (1994), Evolved Virtual Creatures (EVCs) have been a significant area of research. Sims work showed 3D physics simulated creatures were able to produce various complex behaviors. These behaviors included travelling a distance in a physics simulated 3D landscape, following a light source, competing directly against each other in a block ownership task, moving on both land and in water.

After Sims (1994) research, increases in the complexity of the behaviours exhibited by evolved virtual creatures has slowed or stopped to a standstill. Some variations on EVC research had emerged, such as Framsticks (Komosinski. 2000) which involves creatures made of flexible sticks, connected by joints and controlled with neural network. However the physics are simplified and there is limited interaction between agents.

Neural networks were proposed as a method of connecting the sensor inputs of an EVC to their motor outputs. Input signals can include sensors of the surrounding environment such as light distance to objects and perhaps other EVCs. Other sensors can be related to monitoring the EVC's own body to give feedback on motion. 
Many initial EVC models used a fixed topology neural network which was limited is size and complexity which in turn limited behavioral complexity. To avoid this problem, Stanley \& Miikkulainen (2002) proposed the NEAT method which uses a neural network in the same way but applies neuroevolution to enable the brain structure to grow in complexity over time to match requirements of the task by modifying both the weights and topology of links between neurons in the ANN.

\subsection{Related Work}

Recently a few areas of research have begun to exhibit EVCs with a wider range of complex behaviors. There have been developments in the body, the brain controller and resulting behaviour. Robinson et al. (2000) have shown deliberative behaviours on tasks such as river crossing, by combining the evolved ANNs with shunting models and other approaches. This enabled creatures to collect building materials and construct a bridge across the river. However the shunting model could provide the EVC with an internal map of the environment, whereas biological creatures have to discover the map themselves from scratch and memorize the routes which lead to rewards.

Further advances in EVCs producing more complex behavior was produced by the Encapsulation, Syllabus, Pandemonium (ESP) method (Lessin, 2014; Lessin et al. 2014a). Encapsulation captures and separates skills once they have evolved, which prevents them from being un-learnt or forgotten and enables new skills to be learnt without interference. Syllabus is a series of training steps defined by the human to guide the learning process. Pandemonium is preventing learned skills from working against or conflicting with each other. One disadvantage with ESP is that human input is required to define the syllabus.

Conventional EVCs model muscles as uniform drives between rigid sections controlled by a 'brain', however a recent developent implemented muscle density, orientation, attachment points, and size to control the muscles (Lessin et al. 2014b), which helps decrease required complexity.

Rather than humans having to painstakingly design the morphology and control of agile autonomous machines, evolutionary algorithms can be used to search for robotic designs and behaviours without presupposing what those designs and behaviours may be [Cheney et al. 2017]. It can yield machines that do not resemble any animals currently found on earth [Langton et al. 1989].

Recently in 2016 soft bodies model robots were evolved with dual CPPNs [Cheney et al., 2016] one CPPN is for the morphology as a 7x7x7 cube where an output represents presence of material and an output represents type of material. The second CPPN is for control of muscle tissues describing phase offset from a sinusoidal wave input. A limitation to many voxel based soft robot EVC simulations appears to be the limitation of resources, since most currently implement maximum size of 20x20x20 which could limit how meaningful the analogy is between EVCs and biological animal morphology.

Also online simulations of evolving virtual creatures are available (Moore, 2018) and Novelty search has also been applied (Lehman, Stanley 2011). 


\subsection{State of Outstanding Problems}

Legged robots are playing an increasingly important role in our daily lives plus are increasingly used in industry and military. There are commercial consumer walking biped or quadruped robots like Asimo and the dog aibo. They can potentially out perform wheels when on rugged terrain but the disadvantage is the complexity required for their controllers. [Clune et al. 2009b].

Quadruped robot bodies are a popular experiment for evolutionary algorithms. Many scientists report that the algorithms struggle when a large number of parameters need to be configured. They may succeed evolving a controller for one leg but fail once evolution is challenged with evolving a controller from many inputs and outputs from separate legs [Clune et al. 2009b]. This is actually how octopuses work. They have a separate brain to control each leg independently.

Most EVC developments separate the body from the brain. The brain receives input from sensors on the body which as mentioned can sense the environment, other EVCs or parts of the EVC itself such as joint angles. The brain then sends it's decisions to the parts of the body which are required to move. This has resulted in a beautiful and unexpected ability to walk, swim, even move towards a light. Future work could focus on combining brain and body into one, both evolving together.

Future EVC work could aim towards building upon recent advances by Jolley \& Channon (2017) which has used HyperNEAT for deliberative motion planning which can impressively switch between different modes to perform sequences of tasks such as moving back and forth to collect separate stones when building a bridge to cross a river.

Future EVC work should be inspired hybrid neurocontrollers. It was shown by Clune et al. (2009a) that for quadruped gait task HyperNEAT is best used only for the first part of evolution and then it can switch to FTNEAT which is fixed topology to optimise the solution found by HyperNEAT with up to $40 \%$ improvement. This was particularly good at irregular problems where quadruped leg joint angles had an angle of error artificially introduced. This demonstrated that indirect and direct encodings can be used together for the first time, although the advantages of both had been previously noted.

\section{The Machine Learning Task}

The given inputs and expected outputs were kept strictly equal for all tested controllers. Therefore here we can formally define the machine learning task based on the relationship between inputs and outputs of the controller. This is critical step because small changes to the representations of input and output can make big changes to the difficulty of the task for the controller to learn.

In total the EVC has five input sensors: (1) a sine wave which is useful to induce rhythmic motion, (2) 4 join angle sensors. (3) There is one random number input. This is independent so that EVCs can randomly determine an action or movement to take. 


\section{Benchmark Algorithm}

The developed system included designing a custom developed hard-coded benchmark algorithm for generating a forward movement by controlling the angles of the joints directly from the sin wave input.

This results in some forward movement, but is not optimised in any way. This can be used for a comparison between the hard-coded benchmark and the evolved controllers. The benchmark algorithm was used in this research as a comparison or gold standard to assess the performance of the fixed topology and evolving ANN algorithms.

\section{Developed Physics Environment}

A pre-existing 3D simulated physics library called Open Dynamics Engine was used to ensure the complete range of physics interactions were implemented. This was combined with a custom designed C programmed OpenGL Graphics implementation.

An algorithm was created to randomly generate EVCs of various morphology from within a fixed size range. All 4 legs could be different lengths and the body could also be of different sizes and weights. Every generated EVC was a different size. Fig. 1 shows various sizes of EVC which were generated and subsequently simulated in the physics environment, being controlled by random fixed topology ANNs. 


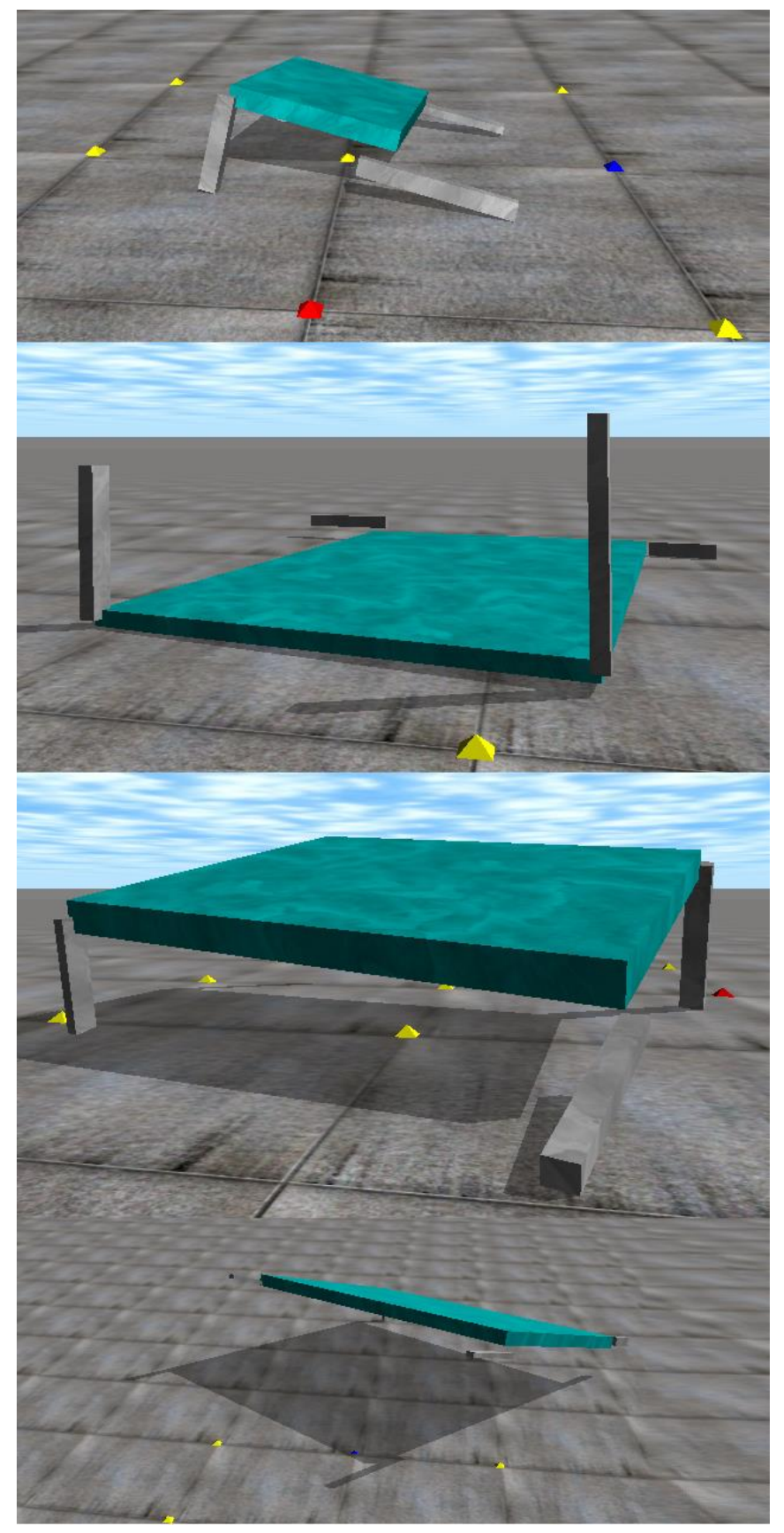

Fig. 1. Various sizes of quadruped EVCs were algorithmically generated with unique ANN con-trollers. One EVC has jumped upside down (2nd from top) and another EVC is in the process of jumping (bottom). A walking strategy evolved (top and 2 nd from bottom). 


\section{$5 \quad$ Fixed Neural Network Training}

Initially a fixed topology ANN was used. The ANN topology had 8 inputs, including 4 joint angle sensors (one for each leg), a sin input, cosine input and two biases. The one hidden layer had 8 fully connected nodes and 4 outputs, with only feed forward connections, no recurrence. The weights were randomly assigned to produce a succession of randomly tuned fixed topology ANNs. This produced some working movement behaviour although it was not producing elegant movement.

In total 5042 randomly generated ANNs were tested. Of these, $4562(90.4 \%)$ travelled less than 5 units. Only 480 ANNs (9.6\%) travelled over 5 units, 86 ANNs (1.7\%) travelled over 10 units, 30 ANNs $(0.5 \%)$ were over 15 units, only 4 ANNs $(0.08 \%)$ travelled more than 20 units. The champion travelled 24.5 units. This distribution is shown in Fig. 2.

This simulation model is interesting since a high performance cannot be obtained only by a well-tuned ANN controller alone, nor only a well-proportioned EVC body, but in fact a combination of these two factors together is required for top performance.

All of the 5042 simulated random EVCs including the best performing were recorded into a comma separated value (csv) file, storing the sizes of all legs and body, plus the values of all ANN connection weights. This feature enables the best performing EVCs to be reloaded on demand into the physics simulator, for examination to identify the features which caused the best EVCs to perform highly.

The best EVCs had an ANN which caused all four legs to move in synchronisation, with the back and front legs moving in a way to produce forward movement. The best EVC bodies was usually long which was more stable and less prone to the problem of flipping over (Fig. 1b).

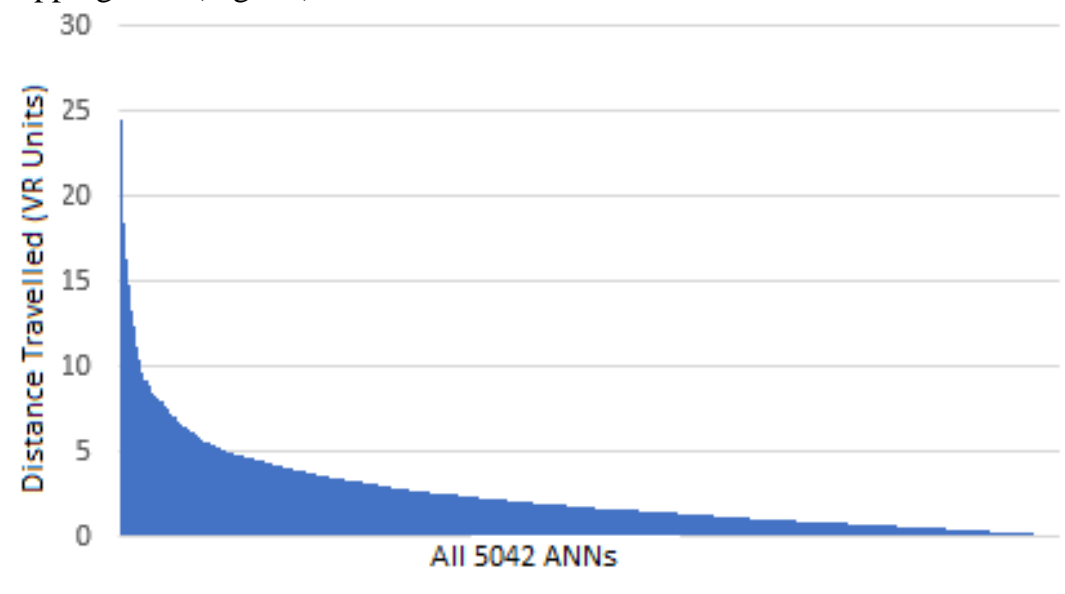

Fig. 2. Assessment of fitness (distance travelled) by 5042 EVCs with randomly generated ANNs, the champion travelled 24.5 units but $90.4 \%$ travelled less than 5 units (ordered by fitness, not by evolutionary generations).

Some of the best and fastest walking behaviors appeared to be very efficient. One technique used a trailing leg to prevent overbalancing. Some techniques used diagonal 
legs in synchronization (similar to galloping) whereas the fastest running techniques used all four legs, including the two front legs together to push forwards (Fig. 3 \& 4).

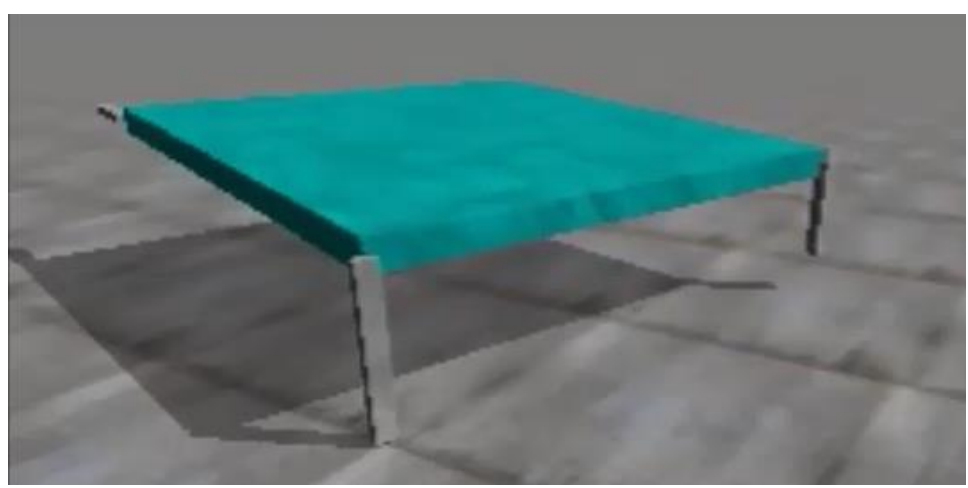

Fig. 3. A running EVC uses a trailing rear leg to prevent overturning.

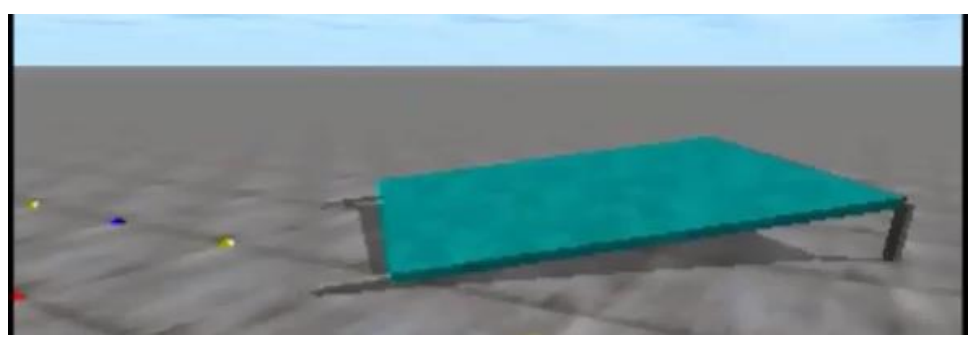

Fig. 4. The EVC uses both front and rear legs together effectively to launch forwards multiple times.

\section{Evolving ANN Controllers}

Neuroevolution was applied to evolve neural networks which were then applied as the controller for EVCs. Between generations, the controller was subject to genetic change, by modifying the ANN both in terms of the weights and the topological structure, including the number and location of connections.

Initially, the ANNs were set blank, with no hidden layer nodes. The additional nodes are added by evolution over time.

The fitness function was set to 1 point for each VR unit of distance travelled from the central starting point.

A comprehensive set of tests was done with EVCs left to run for a fixed length of time. The ANN controllers were subject to neuroevolution in populations of size 15 organisms over 100 generations and this was repeated 10 times. Afterwards a further 5 repetitions of 100 generations was completed, this time with populations of 150 organisms which is a more conventional population size for neuroevolutionary algorithms.

In all runs there were some useful movement strategies evolving. By the 10th generation organisms often had increased their fitness. 
In later experiments the body of the EVC was also subject to genetic change over time, so that an EVC body could evolve to be optimized for the ANN controller, or vice versa.

In the initial experiment, all ANN controllers were tested with the same EVC body shape and size to ensure that furthest movement was due to the ANN and not due to changes in body shape or size.

Initially the most outstanding solution evolved by neuroevolution was to take a large jump in the air (Fig. 5). This satisfied the fitness criteria by travelling a long distance, but often resulted in the EVC landing upside down and it was unable to continue further. Soon it had evolved to land upright so that it could jump a second time. The jumping strategy outperformed walking strategy and became a local optimum as the evolution process tended to select jumping techniques in subsequent generations, preventing further evolution of walking strategies.

The benefits of jumping over walking could also have been influenced by the time that each EVC was left to run before recording it's fitness which was set to a limit of 5 seconds. In this short time, a running or walking EVC may not have had enough time to catch up with a jumping EVC.

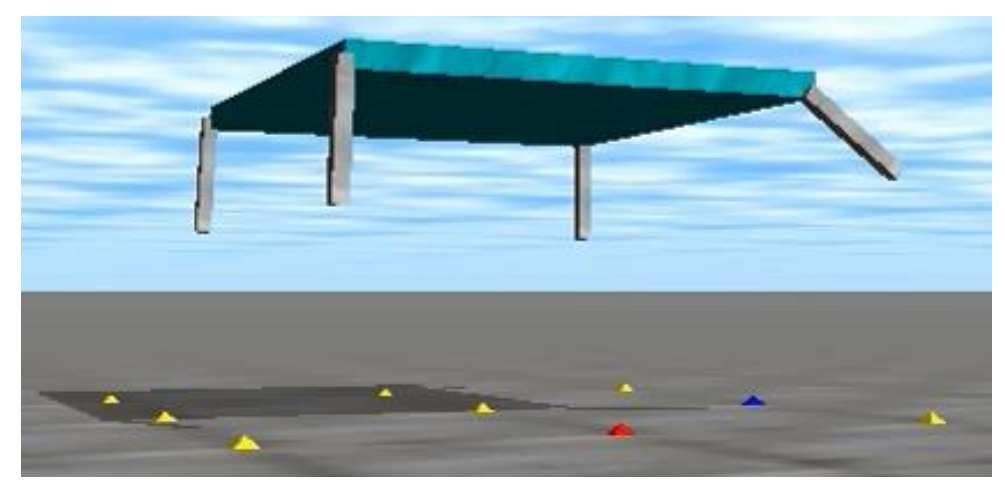

Fig. 5. The neural network controller has evolved a jumping technique, the EVC travels a large distance quickly.

Over time, the fitness increased up to a maximum distance travelled of around 13 VR units, shown in the graph in Fig. 6. Fitness reached various plateaus early on during evolution. After around 1200 EVCs had evolved, the majority of EVCs obtained a fitness of between 8 and 13 .

The distribution of fitness of all EVCs that evolved is shown in Fig. 7. This shows that there are fewer EVCs with fitness below 8, because after the first $1200 \mathrm{EVCs}$, the majority had fitness over 8 . This can be directly compared to the same graph for the fixed topology ANNs (Fig. 2). 


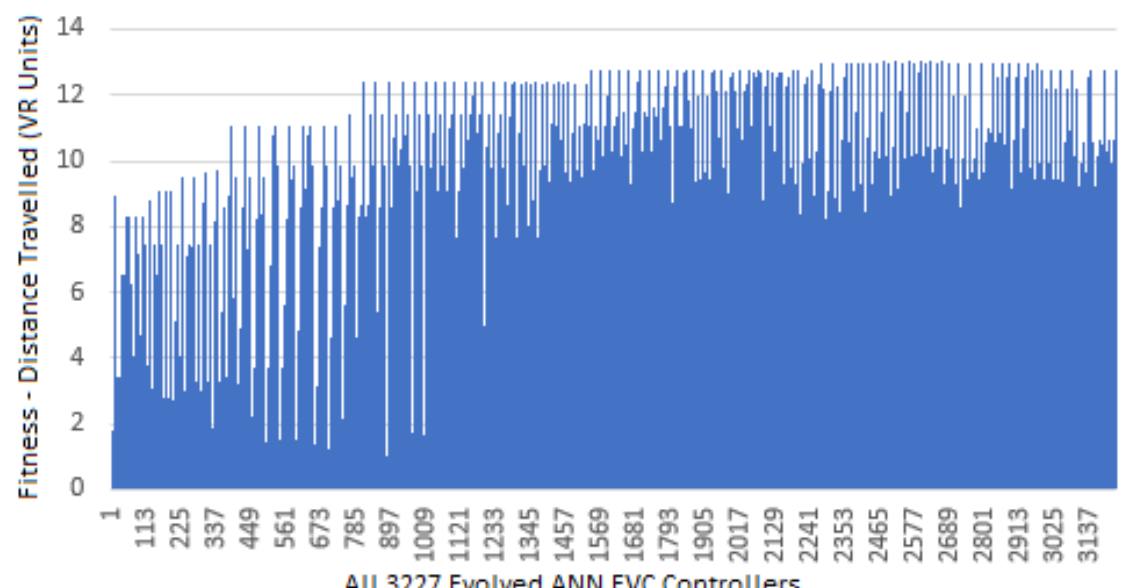

Fig. 4. The fitness over time of each EVC controller during the evolution process (ordered by evolutionary generations).

Neuroevolution produced medium to high fitness much more reliably and often than with fixed ANNs, shown by the comparison between graphs in Figs. 2 and 5. The highest fitness in neuroevolution was less of a chance occurrence and more of a gradual, iterative process reflecting the constructive and selective behavior of natural selection.

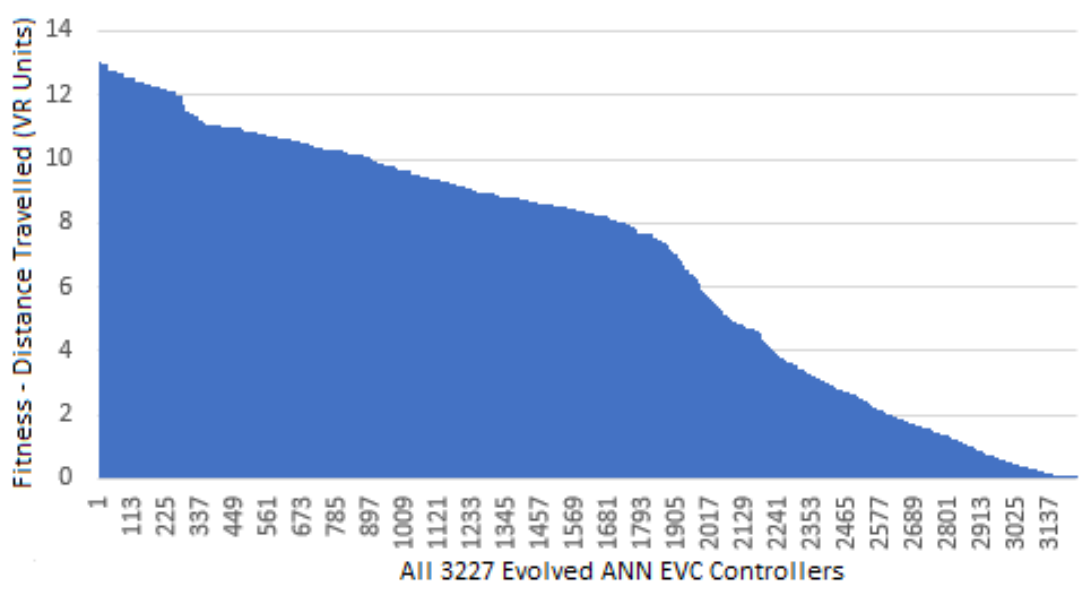

Fig. 5. The fitness of all EVC controllers during the neuroevolutionary process (ordered by fitness).

\section{Conclusions and Future Work}

This paper has investigated the benefits of neuroevolution compared to fixed topology ANN by testing how EVC behaviour can evolve to travel a distance within a physics simulated environment compared to a benchmark algorithm and a fixed ANN. 
This research applied evolutionary algorithms to evolve the ANN topology and weights.

Some of the best and fastest walking behaviors appeared to be very efficient. One technique used a trailing leg to prevent overbalancing. Some techniques used diagonal legs in synchronization (similar to galloping) whereas the fastest running techniques used all four legs, including the two front legs together to push forwards.

Future EVC work could aim towards building deliberative motion planning which can switch between different modes to perform sequences of tasks such as moving back and forth to collect food and return it to a central nest.

Future EVC work should be inspired by hybrid neurocontrollers. For a quadruped gait task such as this, ANN topology evolution could be used only for the first part of evolution and then a fixed topology could be used to optimize the solution found during topology evolution, as this approach was shown by Clune et al. (2009a) to give $40 \%$ improvement. This could be particularly good at irregular problems where quadruped leg joint angles need to overcome obstacles or behave differently in other circumstances. Future work could also investigate indirect and direct encodings being used together during this EVC quadruped gait task.

\section{Acknowledgment}

The research was funded by The Royal Academy of Engineering (RAEng) as part of the Research Fellowship awarded to Dr Neil Vaughan as Principle Investigator.

\section{References}

1. Jolley, B., \& Channon, A. (2017, November). Toward evolving robust, deliberate motion planning with HyperNEAT. In Computational Intelligence (SSCI), 2017 IEEE Symposium Series on (pp. 1-8). IEEE.

2. Lessin, D., Fussell, D., \& Miikkulainen, R. (2014a). Adapting morphology to multiple tasks in evolved virtual creatures. In Proceedings of The Fourteenth International Conference on the Synthesis and Simulation of Living Systems (ALIFE 14) (Vol. 2014, pp. 247-254).

3. Lessin, D., Fussell, D., \& Miikkulainen, R. (2014b). Trading control intelligence for physical intelligence: Muscle drives in evolved virtual creatures. In Proceedings of the 2014 conference on Genetic and evolutionary computation (pp. 705-712). ACM.

4. Lessin, D., (2014), Evolved Virtual Creatures as Content: Increasing Behavioral and Morphological Complexity, Dissertation.

5. Moore J, Evolve-A-Robot, Accessed Online, 24/6/2018, http://jaredmmoore.com/EvoEnv/evo_main.html

6. Lehman, Stanley, (2011), "Evolving a Diversity of Creatures through Novelty Search and Local Competition" Proceedings of the Genetic and Evolutionary Computation Conference (GECCO 2011).

7. M. Komosinski. (2000) The world of framsticks: simulation, evolution, interaction. In Proceedings of 2nd International Conference on Virtual Worlds (VW2000), Paris, pages 214224. Springer-Verlag (LNAI 1834). 
8. Clune, J., Beckmann, B. E., Pennock, R. T., \& Ofria, C. (2009a). HybrID: A hybridization of indirect and direct encodings for evolutionary computation. In European Conference on Artificial Life (pp. 134-141). Springer, Berlin, Heidelberg.

9. Clune, J., Beckmann, B. E., Ofria, C., \& Pennock, R. T. (2009b). Evolving coordinated quadruped gaits with the HyperNEAT generative encoding. In Evolutionary Computation, 2009. CEC'09. IEEE Congress on (pp. 2764-2771). IEEE.

10. Cheney et al. 2016 On the Difficulty of Co-Optimizing Morphology and Control in Evolved Virtual Creatures.

11. Cheney, N., Bongard, J., SunSpiral, V., \& Lipson, H. (2017). Scalable Co-Optimization of Morphology and Control in Embodied Machines. arXiv preprint arXiv:1706.06133.

12. Langton C. G., et al., Artificial life. Addison-Wesley Publishing Company Redwood City, CA, 1989.

13. Robinson, E., Ellis, T., \& Channon, A. (2007). Neuroevolution of agents capable of reactive and deliberative behaviours in novel and dynamic environments. In European Conference on Artificial Life (pp. 345-354). Springer, Berlin, Heidelberg.

14. Sims, K. (1994). Evolving 3D morphology and behavior by competition. Artificial life, 1(4), 353-372.

15. Stanley, K. O., \& Miikkulainen, R. (2002). Evolving neural networks through augmenting topologies. Evolutionary computation, 10(2), 99-127. 